International Journal of Pure and Applied Mathematics

Volume 92 No. 3 2014, 389-401

ISSN: 1311-8080 (printed version); ISSN: 1314-3395 (on-line version)

url: http://www.ijpam.eu

doi: http://dx.doi.org/10.12732/ijpam.v92i3.7

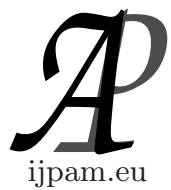

\title{
THE NEGATIVE BINOMIAL-ERLANG DISTRIBUTION WITH APPLICATIONS
}

\author{
Siriporn Kongrod ${ }^{1}$, Winai Bodhisuwan ${ }^{2}$, Prasit Payakkapong ${ }^{3}$ \\ ${ }^{1,2,3}$ Department of Statistics \\ Kasetsart University \\ Chatuchak, Bangkok, 10900, THAILAND
}

\begin{abstract}
This paper introduces a new three-parameter of the mixed negative binomial distribution which is called the negative binomial-Erlang distribution. This distribution obtained by mixing the negative binomial distribution with the Erlang distribution. The negative binomial-Erlang distribution can be used to describe count data with a large number of zeros. The negative binomialexponential is presented as special cases of the negative binomial-Erlang distribution. In addition, we present some properties of the negative binomial-Erlang distribution including factorial moments, mean, variance, skewness and kurtosis. The parameter estimation for negative binomial-Erlang distribution by the maximum likelihood estimation are provided. Applications of the the negative binomial-Erlang distribution are carried out two real count data sets. The result shown that the negative binomial-Erlang distribution is better than fit when compared the Poisson and negative binomial distributions.
\end{abstract}

AMS Subject Classification: 60E05

Key Words: mixed distribution, count data, Erlang distribution, the negative binomial-Erlang distribution, overdispersion

Received: December 4, 2013

(C) 2014 Academic Publications, Ltd. url: www.acadpubl.eu

${ }^{\S}$ Correspondence author 


\section{Introduction}

The Poisson distribution is a discrete probability distribution for the counts of events that occur randomly in a given interval of time, since data in multiple research fields often achieve the Poisson distribution. However, count data often exhibit overdispersion, with a variance larger than the mean. The negative binomial (NB) distribution is employed as a functional from that which relaxes the overdispersion restriction of the Poisson distribution.

The NB distribution is a mixture of Poisson distribution by mixing the Poisson distribution and gamma distribution. The NB distribution has become increasingly popular as a more flexible alternative to count data with overdispersion. The NB distribution was first introduced by Pascal [2]. Montmort [13] applied the NB distribution to represent the number of times that a coin should be flipped to obtain a certain number of heads. Student [16] used the NB distribution as an alternative to the Poisson distribution. Eggenberger and Polya [5] has been shown to be the limiting form of the NB distribution. Ever since, there has been an increasing number of applications of the NB distribution have been developed in a parallel way. For instant, the Poisson-inverse Gaussian distribution [15], [9], the negative binomial-inverse Gaussian distributions [4], [15] and the negative binomial-Beta Exponential distribution [3].

In this paper we introduce a new mixed negative binomial distribution, which is called the negative binomial-Erlang (NB-EL) distribution. The NBEL distribution can be used to describe count data distribution with a large number of zeros in Poisson distribution. The Erlang (EL) distribution was introduced by Agner Erlang [1] was the first author to extend the exponential distribution with his method of stages. He defined a non-negative random variable as the time taken to move through a fixed number of stages, spending an exponential amount of time with a fixed rate in each one.

The NB-EL distribution is obtained by mixing the NB distribution whose probability of success parameter $p$ follows the EL distribution. In other words, if the probability of success parameter $p$ of the NB distribution has the EL distribution, then the resulting distribution is referred to as the NB-EL with parameters $r, k$ and $c$. The negative binomial-exponential has three-parameter is special case of the NB-EL distribution. In particular, various structural properties of the new distribution are derived, including expansions for its factorial moments, mean, variance, skewness and kurtosis. 


\section{The Negative Binomial Distribution}

The negative binomial distribution is a natural and more flexible extension of Poisson distribution and allows for overdispersion relative to the Poisson distribution.

If $X$ is the NB distribution with parameter $r>0$ and $0<p<1$ then we can write the pmf as

$$
f(x ; r, p)=\left(\begin{array}{c}
r+x-1 \\
x
\end{array}\right) p^{r}(1-p)^{x} ; x=0,1,2, \ldots
$$

Note that $p$ is a probability of success, the experiment is repeated as many times as required to obtain $r$ successes.

The first two moments about zero and the factorial moment of order $m$ of the NB distribution are given respectively

$$
\begin{aligned}
& E(X)=\frac{r(1-p)}{p}, \\
& V a r=\frac{r(1-p)}{p^{2}}, \\
& \mu_{[m]}(X)=\frac{\Gamma(r+m)]}{\Gamma(r)}-\frac{(1-p)^{m}}{p^{m}}, \quad m=1,2, \ldots
\end{aligned}
$$

The log likelihood function of the NB distribution is given by

$$
L(r, p)=\prod_{i=1}^{n}\left[\left(\begin{array}{c}
r+x_{i}-1 \\
x_{i}
\end{array}\right) p^{r}(1-p)^{x_{i}}\right]
$$

then, we can write the log likelihood function of the $\mathrm{NB}(r, p)$ as

$$
\begin{aligned}
\log L(r, p) & =\sum_{i=1}^{n} \log \left(\begin{array}{c}
r+x_{i}-1 \\
x_{i}
\end{array}\right)+n r \log (p) \\
& -\log (1-p)\left(\sum_{i=1}^{n} x_{i}\right) .
\end{aligned}
$$




\section{The Erlang Distribution}

The EL distribution was introduced by Agner Erlang [1]. It is a continuous probability distribution with wide applicability mainly due to its relative to the exponential distributions.

The probability density function (pdf) of the EL distribution is given by

$$
g(x)=\frac{c^{k} x^{k-1} e^{-c x}}{(k-1) !},
$$

for $x>0$ and $k, c>0$.

Note that parameter $k$ is called the shape parameter and the parameter $c$ is called the rate parameter.

The mean of the EL distribution is given as

$$
E(X)=\frac{k}{c} .
$$

The variance of the EL distribution is then given as

$$
\operatorname{Var}(X)=\frac{k}{c^{2}} .
$$

The moment generating function of the EL distribution is defined by

$$
M_{X}(t)=\left(1-\frac{t}{c}\right)^{-k} \quad \text { for } \quad t>0 .
$$

\section{The Negative Binomial-Erlang Distribution}

Definition 1. Let $X$ be a random variable of the NB-EL $(r, k, c)$ distribution, when the NB distribution have parameters $\quad r>0$ and $p=\exp (-\lambda)$, where $\lambda$ is distributed as the EL distribution with positive parameters $k$ and $c$, i.e., $X \mid \lambda \sim \mathrm{NB}(r, p=\exp (-\lambda))$ and $\lambda \sim \operatorname{EL}(k, c)$.

Theorem 2. Let $X \sim N B-E L(r, k, c)$. The pmf of $X$ is given by

$$
f(x ; r, k, c)=\left(\begin{array}{c}
r+x-1 \\
x
\end{array}\right) \sum_{j=0}^{x}\left(\begin{array}{l}
x \\
j
\end{array}\right)(-1)^{j}\left(\frac{c}{c+(r+j)}\right)^{k},
$$

for $x>0$ and $k, c>0$. 
Proof. If $X \mid \lambda \sim \mathrm{NB}(r, p=\exp (-\lambda))$ in Eq. 1 and $\lambda \sim \mathrm{EL}(k, c)$ in Eq. 4, then the pmf of $X$ can be obtained by

$$
f(x ; r, k, c)=\int_{0}^{\infty} f_{1}(x \mid \lambda) g(\lambda ; k, c) \mathrm{d} \lambda
$$

where, $f_{1}(x \mid \lambda)$ is defined by

$$
\begin{aligned}
f_{1}(x \mid \lambda) & =\left(\begin{array}{c}
r+x-1 \\
x
\end{array}\right) e^{-\lambda x}\left(1-e^{-\lambda}\right)^{x} \\
& =\left(\begin{array}{c}
r+x-1 \\
x
\end{array}\right) \sum_{j=0}^{x}\left(\begin{array}{l}
x \\
j
\end{array}\right)(-1)^{j} e^{-\lambda(r+j)} .
\end{aligned}
$$

By substituting Eq. 10 into Eq. 9, we obtain

$$
\begin{aligned}
f(x \mid \lambda) & =\left(\begin{array}{c}
r+x-1 \\
x
\end{array}\right) \sum_{j=0}^{x}\left(\begin{array}{l}
x \\
j
\end{array}\right)(-1)^{j}\left(\int_{0}^{\infty} e^{-\lambda(r+j)} f_{2}(\lambda ; \alpha, \beta) \mathrm{d} \lambda\right) \\
& =\left(\begin{array}{c}
r+x-1 \\
x
\end{array}\right) \sum_{j=0}^{x}\left(\begin{array}{l}
x \\
j
\end{array}\right)(-1)^{j}\left(M_{\lambda}(-(r+j))\right) .
\end{aligned}
$$

Substituting the moment generating function of EL distribution Eq. 7 into Eq. 11, the pmf of NB-EL $(r, k, c)$ is finally given as

$$
f(x ; r, k, c)=\left(\begin{array}{c}
r+x-1 \\
x
\end{array}\right) \sum_{j=0}^{x}\left(\begin{array}{l}
x \\
j
\end{array}\right)(-1)^{j}\left(\frac{c}{c+(r+j)}\right)^{k} .
$$

Many well known distributions are subsumed by the NB-EL distribution. Next, we display graphs of the pmf of the NB distribution with various values of parameters are shown in Figure 1.

Corollary 3. If $k=1$ then the NB-EL distribution reduces to the negative binomial-exponential (NB-E) distribution with pmf given by

$$
f(x ; r, c)=\left(\begin{array}{c}
r+x-1 \\
x
\end{array}\right) \sum_{j=0}^{x}\left(\begin{array}{l}
x \\
j
\end{array}\right)(-1)^{j}\left(\frac{c+(r+j)}{c}\right),
$$

where $x=0,1,2, \ldots$ for $r$ and $c>0$.

Proof. If $X \mid \lambda \sim \operatorname{NB}(r, p=\exp -\lambda)$ and $\lambda \sim \operatorname{EL}(k=1, c)$, then the pmf of $X$ is

$$
f(x ; r, c)=\left(\begin{array}{c}
r+x-1 \\
x
\end{array}\right) \sum_{j=0}^{x}\left(\begin{array}{l}
x \\
j
\end{array}\right)(-1)^{j}\left(\frac{c+(r+j)}{c}\right)
$$


(a)

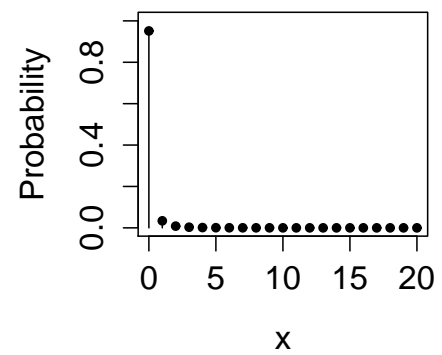

(c)

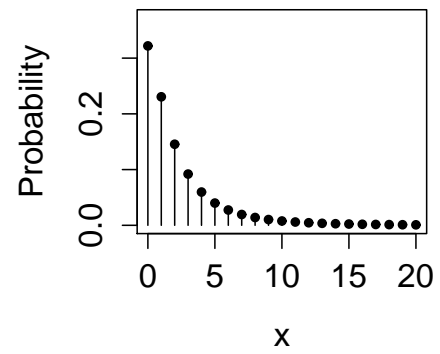

(e)

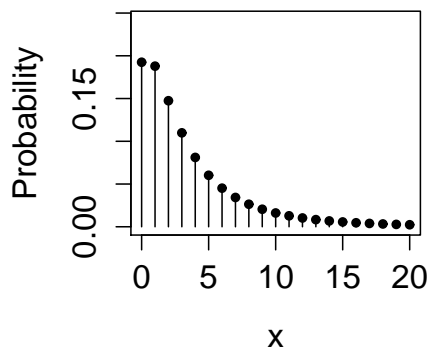

(b)

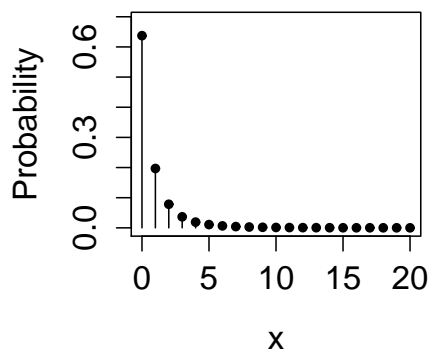

(d)

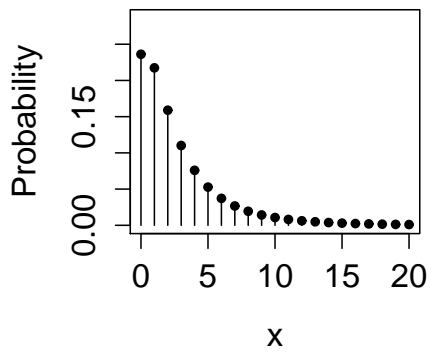

(f)

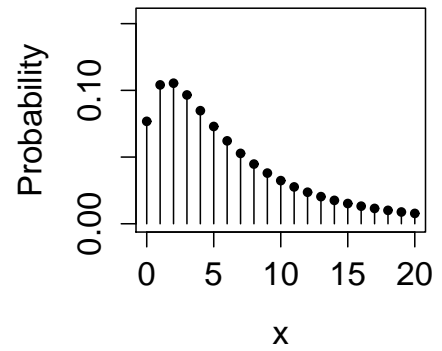

Figure 1: (a-f): The probability mass function of the NB-EL distribution of some values of parameters: (a) $\mathrm{r}=0.5, k=0.5, c=0.5$, $\mathrm{r}=0.5, k=1, c=0.5,(\mathrm{c}) \mathrm{r}=0.5, k=2, c=0.5$, (d) $\mathrm{r}=5, k=1, c=1.5,(\mathrm{e}) \mathrm{r}=5$, $k=1, c=2$ and (f) $\mathrm{r}=5, k=2, c=5$ 


$$
=\left(\begin{array}{c}
r+x-1 \\
x
\end{array}\right) \sum_{j=0}^{x}\left(\begin{array}{l}
x \\
j
\end{array}\right)(-1)^{j}\left(\frac{c+(r+j)}{c}\right) .
$$

From Corollary 3, we find the negative binomial-exponential distribution displayed in Eq. 12, which introduced by Panger and Willmot [17].

\section{Properties of the NB-EL Distribution}

In this section, we have studied the properties of the NB-EL distribution, which includes the factorial moments, mean, variance, skewness and kurtosis are given as follow

Theorem 4. If $X \sim N B-E L(r, k, c)$, then the factorial moment of order $m$ of $X$ is given by

$$
\mu_{[m]}(X)=\frac{\Gamma(r+m)}{\Gamma(r)} \sum_{j=0}^{m}\left(\begin{array}{c}
m \\
j
\end{array}\right)(-1)^{j}\left(\frac{c}{c-(m-j)}\right)^{k},
$$

where $x=0,1,2, \ldots$ for $r, k$ and $c>0$.

Proof. If $X \mid \lambda \sim \mathrm{NB}(r, p=\exp (-\lambda))$ in Eq. 1 and $\lambda \sim \operatorname{EL}(k, c)$ in Eq. 4, then the factorial moment of order $m$ of $X$ can be obtained by

$$
\mu_{[k]}(X)=E_{\lambda}\left[\mu_{k}(X \mid \lambda)\right] .
$$

The factorial moment of order $m$ of the NB distribution in Eq. 2, $\mu_{[m]}(X)$ becomes

$$
\mu_{[m]}(X)=E_{\lambda}\left(\frac{\Gamma(r+m)}{\Gamma(r)} \frac{\left(1-e^{-\lambda}\right)^{m}}{e^{-\lambda m}}\right)=\frac{\Gamma(r+m)}{\Gamma(r)} E_{\lambda}\left(e^{\lambda}-1\right)^{m} .
$$

Using a binomial expansion of $\left(e^{\lambda}-1\right)^{m}$, then shows that $\mu_{[m]}(X)$ can be written as

$$
\begin{aligned}
\mu_{[m]}(X) & =\frac{\Gamma(r+m)}{\Gamma(r)} \sum_{j=0}^{m}\left(\begin{array}{c}
m \\
j
\end{array}\right)(-1)^{j} E_{\lambda}\left(e^{\lambda(m-j)}\right) \\
& =\frac{\Gamma(r+m)}{\Gamma(r)} \sum_{j=0}^{m}\left(\begin{array}{c}
m \\
j
\end{array}\right)(-1)^{j} M_{\lambda}(m-j) .
\end{aligned}
$$


From the moment generating function of the EL distribution in Eq. 7, we have finally that $\mu_{[m]}(X)$ can be written as

$$
\mu_{[m]}(X)=\frac{\Gamma(r+m)}{\Gamma(r)} \sum_{j=0}^{m}\left(\begin{array}{c}
m \\
j
\end{array}\right)(-1)^{j}\left(\frac{c}{c-(m-j)}\right)^{k}
$$

From the factorial moments of the NB-EL distribution, it is straightforward to reduce the first four moments given in Eq. 14 - Eq. 17, variance in Eq. 18, skewness in Eq. 19 and kurtosis in Eq. 20.

$$
\begin{aligned}
E(X)= & r\left(\varphi_{1}-1\right) \\
E\left(X^{2}\right)= & \left(r^{2}+r\right) \varphi_{2}-\left(2 r^{2}+r\right) \varphi_{1}+r^{2}, \\
E\left(X^{3}\right)= & \left(r^{3}+3 r^{2}+2 r\right) \varphi_{3}-\left(3 r^{3}+6 r^{2}+3 r\right) \varphi_{2} \\
& +\left(3 r^{3}+3 r^{2}+r\right) \varphi_{1}-r^{3}, \\
E\left(X^{4}\right)= & \left(r^{4}+6 r^{3}+11 r^{2}+6 r\right) \varphi_{4}-\left(4 r^{4}+18 r^{3}+26 r^{2}+12 r\right) \varphi_{3} \\
& +\left(6 r^{4}+18 r^{3}+19 r^{2}+7 r\right) \varphi_{2} \\
& -\left(4 r^{4}+6 r^{3}+4 r^{2}+r\right) \varphi_{1}+r^{4},
\end{aligned}
$$

$$
\begin{aligned}
\operatorname{Var}(X) & =E\left(X^{2}\right)-(E(X))^{2} \\
& =\left(r^{2}+r\right) \varphi_{2}-r \varphi_{1}\left(1+r \varphi_{1}\right)
\end{aligned}
$$

$$
\begin{aligned}
\operatorname{Skewness}(X)= & {\left[E\left(X^{3}\right)-3 E\left(X^{2}\right) E(X)+2[E(X)]^{3} /\right] \sigma^{3} } \\
= & {\left[\left(r^{3}+3 r^{2}+2 r\right) \varphi_{3}-\left(3 r^{2}+3 r\right) \varphi_{2}+r \varphi_{1}+3 r^{2}\left(\varphi_{2}\right)^{2}\right.} \\
& \left.-\left(3 r^{3}+3 r^{2}\right) \varphi_{1} \varphi_{2}+2 r^{3}\left(\varphi_{2}\right)^{3}\right] / \sigma^{3}
\end{aligned}
$$

$$
\begin{aligned}
\operatorname{Kurtosis}(X)= & {\left[E\left(X^{4}\right)-4 E\left(X^{3}\right) E(X)+6 E\left(X^{2}\right)(E(X))^{2}-3[E(X)]^{4} /\right] \sigma^{4} } \\
= & {\left[\left(r^{4}+6 r^{3}+11 r^{2}+6 r\right) \varphi_{4}-\left(6 r^{3}+18 r^{2}+12 r\right) \varphi_{3}-3 r^{4}\left(\varphi_{1}\right)^{4}\right.} \\
& +\left(7 r^{2}+7 r\right) \varphi_{2}-4 r \varphi_{1}-6 r^{3}\left(\varphi_{1}\right)^{3}+\left(12 r^{3}+12 r^{2}\right) \varphi_{1} \varphi_{2} \\
& \left.-\left(4 r^{4}+12 r^{3}+8 r^{2}\right) \varphi_{1} \varphi_{3}+\left(6 r^{4}+6 r^{3}\right)\left(\varphi_{1}\right)^{2} \varphi_{2}\right] / \sigma^{4},
\end{aligned}
$$


where

$$
\varphi_{i}=\left(\frac{c-i}{c}\right)^{-k}
$$

\section{Parameters Estimation}

The estimation of parameters for NB-EL distribution via the maximum-likelihood estimation (MLE) method procedure will be discussed. The likelihood function of the NB-EL $(r, k, c)$ is given by

$$
L(r, k, c)=\prod_{i=1}^{n}\left(\begin{array}{c}
r+x_{i}-1 \\
x_{i}
\end{array}\right) \sum_{j=0}^{x_{\mathrm{i}}}\left(\begin{array}{c}
x_{i} \\
j
\end{array}\right)(-1)^{j}\left(\frac{c}{c+(r+j)}\right)^{k} .
$$

with corresponding log-likelihood function

$$
\begin{aligned}
\mathcal{L} & =\log L(r, k, c)=\sum_{i=1}^{n} \log \left(\begin{array}{c}
r+x_{i}-1 \\
x_{i}
\end{array}\right) \\
& +\sum_{i=1}^{n}\left(\log \sum_{j=0}^{x_{i}}\left(\begin{array}{c}
x_{i} \\
j
\end{array}\right)(-1)^{j}\left(\frac{c}{c+(r+j)}\right)^{k} j .\right.
\end{aligned}
$$

The first order conditions for finding the optimal values of the parameters obtained by differentiating Eq. 22 with respect to $r, k$ and $c$ give rise to the following differential equations

$$
\begin{aligned}
& \frac{\partial \mathcal{L}}{\partial r}=\sum_{i=1}^{n} \sum_{k=0}^{x_{\mathrm{i}}-1} \log (r+k) \\
&+\sum_{i=1}^{n}\left\{\frac{-k \sum_{j=0}^{x_{\mathrm{i}}}\left(\begin{array}{c}
x_{\mathrm{i}} \\
j
\end{array}\right)(-1)^{j} \frac{c^{\mathrm{k}}}{(c+(r+j))^{\mathrm{k}+1}}}{\sum_{j=0}^{x_{\mathrm{i}}}\left(\begin{array}{c}
x_{\mathrm{i}} \\
j
\end{array}\right)(-1)^{j}\left[\frac{c}{c+(r+j)}\right]^{k}}\right\}, \\
& \frac{\partial \mathcal{L}}{\partial k}=\sum_{i=1}^{n}\left\{\frac{\sum_{j=0}^{x_{\mathrm{i}}}\left(\begin{array}{c}
x_{\mathrm{i}} \\
j
\end{array}\right)(-1)^{j}\left[\frac{c}{c+(r+j)}\right]^{k} \log \left[\frac{c}{c+(r+j)}\right]}{\sum_{j=0}^{x_{\mathrm{i}}}\left(\begin{array}{c}
x_{\mathrm{i}} \\
j
\end{array}\right)(-1)^{j}\left[\frac{c}{c+(r+j)}\right]^{k}}\right\},
\end{aligned}
$$

and

$$
\frac{\partial \mathcal{L}}{\partial r}=\sum_{i=1}^{n}\left\{\frac{\sum_{j=0}^{x_{\mathrm{i}}}\left(\begin{array}{c}
x_{\mathrm{i}} \\
j
\end{array}\right)(-1)^{j}\left[\frac{k c^{\mathrm{k}-1}(r+j)}{(c+r+j)^{\mathrm{k}+1}}\right]}{\sum_{j=0}^{x_{\mathrm{i}}}\left(\begin{array}{c}
x_{\mathrm{i}} \\
j
\end{array}\right)(-1)^{j}\left[\frac{c}{c+(r+j)}\right]^{k}}\right\} .
$$


We estimated parameters of the NB-EL distribution with equating Eq. 23 Eq. 25 to zero, the MLE solutions of $\hat{r}, \hat{k}$ and $\hat{c}$ can be obtained by solving the resulting equations simultaneously using a numerical procedure, the NewtonRaphson method.

\section{Illustrative Examples}

The NB-EL distribution is applied on two real count data sets. The details are presented in the following examples.

Example 1. We use a real data set is numbers of injured from the accident on major road in Bangkok in 2007 [3]. The data was collected by Department of Highways, Ministry of Transport, Thailand. We use the real data are fitted by the Poisson, NB and NB-EL distributions (Table 1), which show the observed and expected frequencies, grouped in classes of expected frequency greater than five for the chi-square goodness of fit test. Based on the $p$-value, the maximum likelihood method provides very poor fit for the Poisson and the NB distribution. We can see that NB-EL distribution provides the highest $p$-value of fitting for this data set.

Table 1: Observed and expected frequencies for the accident data

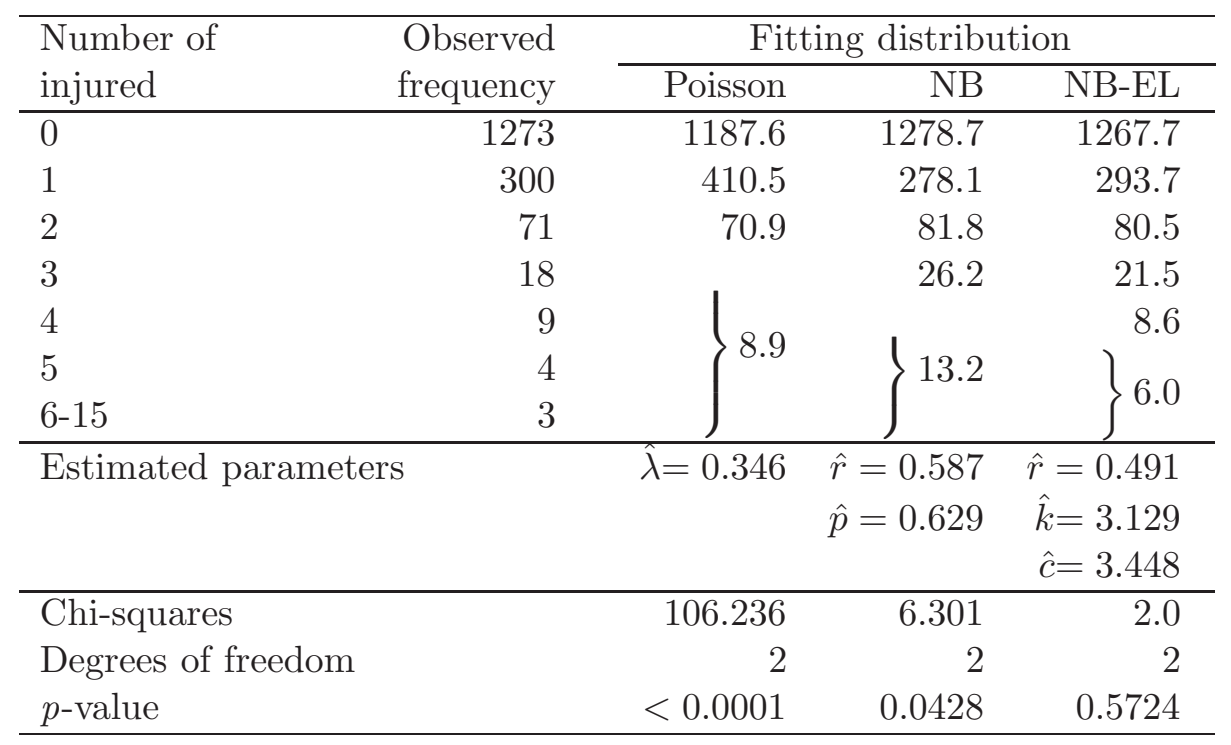

Example 2. We used a data set which was obtained from Klugman et 
al. [15], provides the data set of 9,461 automobile insurance policies where by the number of accidents of each policy has been recorded. We apply this data set to fit with the Poisson, NB and NB-EL distributions. Obtained results (Table 2), shows that the observed and expected frequencies, grouped in classes of expected frequency greater than five for the chi-square goodness of fit test. The maximum likelihood method provides very poor fit for the Poisson and the NB distribution. Based on $p$-value we found that the NB-EL distribution seen to be best fit among there three distributions, with $p$-value 0.890 .

Table 2: Observed and expected frequencies for the accident data

\begin{tabular}{|c|c|c|c|c|}
\hline \multirow{2}{*}{$\begin{array}{l}\text { Number of } \\
\text { claims }\end{array}$} & \multirow{2}{*}{$\begin{array}{l}\text { Observed } \\
\text { frequency }\end{array}$} & \multicolumn{3}{|c|}{ Fitting distribution } \\
\hline & & Poisson & NB & NB-EL \\
\hline 0 & 7840 & 7638.3 & 7841.2 & 7834.6 \\
\hline 1 & 1317 & 1634.6 & 1291.7 & 1306.3 \\
\hline 2 & 239 & 174.9 & 258.1 & 253.4 \\
\hline 3 & 42 & \multirow{6}{*}{13.2} & 54.6 & 42.8 \\
\hline 4 & 14 & & \multirow{5}{*}{15.4} & 15.5 \\
\hline 5 & 4 & & & \\
\hline 6 & 4 & & & \\
\hline 7 & 1 & & & 0.0 \\
\hline $8+$ & 0 & & & \\
\hline \multirow{3}{*}{\multicolumn{2}{|c|}{ Estimated parameters }} & $\hat{\lambda}=0.214$ & $\hat{r}=0.701$ & $\hat{r}=0.311$ \\
\hline & & & $\hat{p}=0.765$ & $\hat{k}=3.428$ \\
\hline & & & & $\hat{c}=5.127$ \\
\hline \multicolumn{2}{|l|}{ Chi-squares } & 293.803 & 8.657 & 1.094 \\
\hline \multirow{2}{*}{\multicolumn{2}{|c|}{$\begin{array}{l}\text { Degrees of freedom } \\
p \text {-value }\end{array}$}} & 2 & 2 & 2 \\
\hline & & $<0.0001$ & 0.014 & 0.890 \\
\hline
\end{tabular}

\section{Conclusion}

In this paper, we introduce a new three-parameter negative binomial-Erlang distribution, NB-EL $(r, k, c)$. This distribution obtained by mixing the NB with the EL distribution (when the NB distribution have parameters $r>0$ and $p=\exp (-\lambda)$, where $\lambda$ is distributed as the EL distribution with positive parameters $k$ and $c$ ). We showed that the negative binomial-exponential distributions is a special case of the NB-EL distribution. In addition, the moments of the NBEL distribution which includes the factorial moments, mean, variance, skewness 
and kurtosis are derived. Moreover, the parameter estimation of the NB-EL using the maximum likelihood estimation are developed. We include an application of the NB-EL distribution to fit two real data sets. We found that this data set is best fit with NB-EL distribution among there three distributions. We hope that NB-EL distribution may attract wider applications in analyzing count data.

\section{Acknowledgments}

We would like to thank Dr.Chookait Pudprommarat, who has given many useful comments and valuable suggestions. This work was supported by research funding from the Research Professional Development Project under the Science Achievement Scholarship of Thailand (SAST).

\section{References}

[1] A.K. Erlang, Solution of some problems in the theory of probabilities of significance in automatic telephone exchanges, Elektrotkeknikeren, 13(1917), 513.

[2] Blaise Pascal, Varia opera mathematica D.Petri de Fermat, Tolossae, (1679).

[3] C. Pudprommarat, W. Bodhisuwan, A new mixed negative binomial distribution, Journal of Applied Sciences, 12(2012), 1853-1858, doi: 10.3923/jas.2012.1853.1858.

[4] E. Gomez-Deniz, J.M. Sarabia and E. Calderin-Ojeda, Univariate and multivariate versions of the negative binomial-inverse Gaussian distributions with applications, Insurance: Mathematics and Economics, 4(2008), doi: 39-49.10.1016/J.insmatheco.2006.12.001.

[5] F. Eggenberger and G.Polya, Uber die Statistik verketetter Votgange, Zeitschrift fur Angewandte Mathematik and Mechanik, 1 (1923), 279-289, doi: $10.1002 /$ zamm.19230030407.

[6] G.E. Willmot, The Poisson-inverse Gaussian distribution as an alternative to the negative binomial, Scandinavian Actuar, 2 (1987), 113-127, doi: 10.1080/03461238.1987.10413823 
[7] H. Ranger and E. Willmot, Finite sum evaluation of the negative binomial exponential model, Astin Bulletin, 12(1981), 133-137.

[8] J. Lemaire, How to define a bonus-malus system with an exponential utility function. ASTIN Bull. 10(1979), 274-282.

[9] L. Tremblay, Using the Poisson inverse Gaussian in bonus-malus systems, ASTIN Bull, 22(1992), 97-106.

[10] L.J. Simon, Fitting negative binomial distributions by the method of maximum likelihood, Proc. Casual. Actuar. Soc., 48(1961), 45-53.

[11] M. Greenwood and G.U. Yule, An inquiry into the Nature of frequency distributions representative of multiple happenings with particular reference to the occurrence of multiple attacks of disease or of repeated accidents, Journal of the Royal Statistical Society, 83(1920), 255-279.

[12] M.D. Bressoud, A radical approach to real analysis, Cambridge University Press, Cambridge (2005).

[13] P.R. de Montmort, Essay d'Analyse sur les Jeux de Hazard, Seconde Edition, Revue Augmentee de plusieurs Lettre, Quillau, Paris (1980).

[14] S. Meng,Y. Wei and G.A. Whitmore, Accounting for individual overdispersion in a bonus-malus system, ASTIN Bull.(1999).

[15] S.A. Klugman. H.H. Panjer and G.E.Willmot, Loss Models: From Data to Decision, John Wiley and Sons USA, 101-159 (2008).

[16] W.S. Gosset Student, On the error of counting with a Haemacytometer, Biometrika, 5(1907), 351-360.

[17] H.H. Panjer and G.E. Willmot, Finite sum eqvluation of the negative binomail-exponential model, Astin Bulletin, 12(1981), 133-137. 
